\title{
Differential expression of cell adhesion molecules in an ionizing radiation-induced breast cancer model system
}

\author{
GLORIA M. CALAF ${ }^{1,2}$, DEBASISH ROY ${ }^{3}$, GOPESHWAR NARAYAN $^{4}$ and ADAYABALAM S. BALAJEE ${ }^{2}$ \\ ${ }^{1}$ Institute for Advanced Research, Tarapacá University, Arica, Chile; ${ }^{2}$ Center for Radiological Research, \\ Columbia University Medical Center, New York, NY 10032; ${ }^{3}$ Department of Natural Sciences, Hostos College \\ of the City University of New York, Bronx, NY 10451, USA; ${ }^{4}$ Department of Human Molecular Genetics, \\ Banaras Hindu University, Varanasi 221005, India
}

Received March 4, 2013; Accepted April 2, 2013

DOI: $10.3892 /$ or.2013.2448

\begin{abstract}
Cell-cell adhesion is mediated by members of the cadherin-catenin system and among them E-cadherin and $\beta$-catenin are important adhesion molecules for epithelial cell function and preservation of tissue integrity. To investigate the importance of cell adhesion molecules in breast carcinogenesis, we developed an in vitro breast cancer model system wherein immortalized human breast epithelial cell line, MCF-10F, was malignantly transformed by exposure to low doses of high linear energy transfer (LET) $\alpha$ particle radiation $(150 \mathrm{keV} /$ $\mu \mathrm{m})$ and subsequent growth in the presence or absence of $17 \beta$-estradiol. This model consisted of human breast epithelial cells in different stages of transformation: i) parental cell line MCF-10F; ii) MCF-10F continuously grown with estradiol at $10^{-8}$ (Estrogen); iii) a non-malignant cell line (Alpha3); and iv) a malignant and tumorigenic cell line (Alpha5) and the Tumor2 cell line derived from the nude mouse xenograft of the Alpha5 cell line. Expression levels of important cell adhesion molecules such as $\alpha$-catenin, $\beta$-catenin, $\gamma$-catenin, E-cadherin and integrin were found to be higher at the protein level in the Alpha5 and Tumor 2 cell lines relative to these levels in the non-tumorigenic MCF-10F, Estrogen and Alpha3 cell lines. In corroboration, cDNA expression analysis revealed elevated levels of genes involved in the cell adhesion function [E-cadherin, integrin $\beta 6$ and desmocollin3 (DSc3)] in the Alpha5 and Tumor2 cell lines relative to the levels in the MCF-10F, Estrogen and Alpha3 cell lines. Collectively, our results suggest that cell adhesion molecules are expressed at higher levels in malignantly transformed breast epithelial cells relative to levels in non-malignant cells. However, reduced levels of adhesion molecules observed in the mouse xenograft-derived Tumor 2 cell line compared to the pre-tumorigenic Alpha5 cell line suggests that the altered
\end{abstract}

Correspondence to: Dr Gloria M. Calaf, Institute for Advanced Research, Tarapacá University, Calle Antofagasta \#1520, Arica, Chile

E-mail: gmc24@columbia.edu

Key words: biomarkers, cell adhesion, estrogen, radiation, breast cells expression levels of adhesion molecules depend on the tumor tissue microenvironment.

\section{Introduction}

Cell-cell adhesion is mediated by members of the cadherincatenin system and among them E-cadherin and $\beta$-catenin are important adhesion molecules involved in the viability and function of epithelial cells as well as tissue integrity (1-10). $\beta$-catenin, $\alpha$-catenin and $\gamma$-catenin are proteins that bind to the highly conserved intracellular cytoplasmatic tail of E-cadherin $(1,10)$. $\beta$-catenin, a $92-\mathrm{kDa}$ protein, has been found to be associated with the cytoplasmic portion of E-cadherin and this association is critical for cell adhesion (4). Differential expression of $\beta$-catenin has been reported in human cancers (7). Loss of E-cadherin- $\beta$-catenin adhesion is an important step in the progression of many epithelial malignancies. The function of the cadherin-catenin system in cell adhesion as well as in intracellular signaling appears to be regulated by multiple factors and by different molecular mechanisms $(1,3,4,9)$. E-cadherin belongs to the family of cell adhesion molecules that are $\mathrm{Ca}^{2+}$-dependent $(5,6) . \beta$-catenin regulates the function of cadherin in cell-to-cell adhesion which is critical for the maintenance of tissue structure and morphogenesis. The intracellular domain of E-cadherin interacts with a variety of cytoplasmic proteins such as $\beta$-catenin, $\alpha$-catenin and $\alpha$-actinin.

Interaction of $\alpha$-actinin with the cadherin/catenin cellcell adhesion complex has been observed via $\alpha$-catenin (4). $\alpha$-catenin, a $102-\mathrm{kDa}$ protein, was initially described as an E-cadherin-associated molecule (10), but it has been shown to be associated with other members of the cadherin family, such as $\mathrm{N}$-cadherin and $\mathrm{P}$-cadherin $(1,5,6,8)$. It has crucial functions in the E-cadherin-mediated cell-cell adhesion system and also as a downstream signaling molecule in the Wnt pathway (5). $\gamma$-catenin, an $82-\mathrm{kDa}$ protein, also known as the fourth armadillo repeat of plakoglobin, is associated with high affinity binding to the cytoplasmic domains of E-cadherin and desmosomal cadherin DSg2. It also binds with $\alpha$-catenin and $\mathrm{N}$-catenin (2).

Integrins are not considered to be bona fide target molecules for oncogenes or tumor suppressors, yet their expression 
levels appear to be altered by transformation in breast cancer cells $(11,12)$. No characteristic integrin expression pattern can be ascribed to all breast tumors and it is likely that several subtypes of breast cancer may generate tumors with a distinct expression pattern of integrins (13). Alteration of integrin expression in the breast can be regarded as a marker of pre-malignant origin (13). The markers used to characterize cell lines include integrin receptors, which are cell adhesion molecules that primarily mediate cell-matrix interactions, being localized to focal contacts, or in the case of the $\alpha-6$ $\beta$-4 integrin heterodimer to hemidesmosomes (14). High expression level of $\alpha-6$ integrin in human breast carcinomas correlates with poor prognosis (14). The prognostic value of $\alpha-6 \beta-4$ integrin expression in breast carcinomas is influenced by laminin production from tumor cells (11).

Cell-cell adhesion molecules including E-cadherin are identified in adherent junctions whereas desmocollin (DSc) glycoproteins are localized in desmosomes. Biological markers used to differentiate between cell phenotypes also revealed components of desmosomes such as desmocollin 1-3 (DSc). These cell adhesion molecules are transmembrane proteins of the cadherin family that form the adhesive core of desmosomes. Desmosomes are sites of adhesion between adjacent cells in layers of epithelia, as well as in certain non-epithelial tissues, and play an important role in the maintenance of tissue architecture. DSc3 which is an important glycoprotein and active member of the cadherin superfamily of calcium-dependent cell-cell adhesion molecules and a principle component of desmosomes plays a pivotal role in maintaining tissue architecture; and therefore, loss of this component leads to a lack of adhesion and a gain in cellular motility. DSc3 expression is usually downregulated in breast cancer cell lines and in primary breast tumors.

In vitro model systems have been extensively used to gain insights into the molecular events of cancer initiation and promotion and to identify novel prognostic/diagnostic markers for various types of cancer. The human breast epithelial cell line MCF-10F, spontaneously immortalized and derived from the breast tissue of a 36-year-old female, has the morphological characteristics of normal breast epithelial cells (15-18). The MCF-10F cell line has been used to detect sensitivity to both chemical carcinogens such as 7, 12, dimethylbenz(a)anthracene (DMBA) and benzo(a)pyrene (BP) (15) and environmental carcinogens such as ionizing radiation (16). It was previously shown that estrogen was a prerequisite for the process of high linear energy transfer (LET) radiation-induced carcinogenesis (16). Several phenotypic properties such as growth rate, anchorage-independent growth and invasive characteristics have also been reported to be grossly similar during the transformation process induced by chemical carcinogens (19-25) and environmental factors, e.g., ionizing radiation (16). The chemo-invasion or the ability of transformed cells to infiltrate the basement membrane in vitro was correlated well with the in vivo malignant characteristics. A Previous study demonstrated that cell adhesion molecules are highly altered in malignantly transformed cells relative to non-tumorigenic cell lines indicating that their altered expression may support or promote breast carcinogenic events (25). Results from our laboratory also found that a combination of estrogen and the organophosphorous compound parathion increased the expression of certain adhesion molecules such as CD146 and $\beta$-catenin and the combined treatment was found capable of altering cell proliferation and inducing transformation of the MCF-10F cell line. To understand the importance of cell adhesion molecules in carcinogenic events, we aimed to examine their expression at different stages of our breast cancer model system developed by the combined treatment of high LET radiation and estrogen.

\section{Materials and methods}

Cell lines. MCF-10F cells were grown in DMEM/F-12 (1:1) medium supplemented with antibiotics $[100 \mathrm{U} / \mathrm{ml}$ penicillin, $100 \mu \mathrm{g} / \mathrm{ml}$ streptomycin, $2.5 \mu \mathrm{g} / \mathrm{ml}$ amphotericin B (all from Life Technologies, Grand Island, NY, USA)] and $10 \mu \mathrm{g} / \mathrm{ml}$ and $5 \%$ equine serum (Biofluids, Rockville, MD, USA), $0.5 \mu \mathrm{g} / \mathrm{ml}$ hydrocortisone (Sigma, St. Louis, MO, USA) and $0.02 \mu \mathrm{g} / \mathrm{ml}$ epidermal growth factor (Collaborative Research, Bedford, MA, USA) were added (15-25). An in vitro experimental breast cancer model (Alpha model) developed by exposure of the immortalized human breast epithelial cell line, MCF-10F, to low doses of high LET $\alpha$ particle radiation $(150 \mathrm{keV} / \mu \mathrm{m})$ and subsequent growth in the presence or absence of $17 \beta$-estradiol was used in this study. This model consisted of human breast epithelial cells at different stages of transformation: i) a control cell line (MCF-10F); ii) MCF-10F continually treated with estradiol at $10^{-8} \mathrm{M}$ (E or Estrogen) (Sigma-Aldrich) named Estrogen cell line; iii) a malignant cell line (Alpha3); and iv) a malignant and tumorigenic cell line (Alpha5) and the Tumor2 cell line derived from cells originating from a tumor after injection of Alpha5 cells in nude mice.

Protein expression by immunocytochemistry. Exponentially growing cells were plated on a glass chamber slide (Nunc Inc., Naperville, IL, USA) as previously described (16) at a density of $1 \times 10^{4}$ cells $/ \mathrm{ml}$ of growth medium. Three independent biological experiments were performed. The following primary antibodies were used for detecting the protein expression: $\beta$-catenin (E-5) (Sc-7963), $\alpha$-catenin E (G-11) (Sc-9988) and $\gamma$-catenin (H-1) (Sc-5415); E-cadherin (N-20) (Sc-1500) and integrin $\beta 3$ (N-20) (Sc-6627) (all from Santa Cruz Biotechnology, Santa Cruz, CA, USA). Rhodamine conjugated secondary antibody was from Jackson ImmunoResearch Laboratories (West Grove, PA, USA). All the antibodies were used at a 1:500 dilution from the original stock concentrations. Slides were mounted with coverslips using Vectashield mounting medium (Vector Laboratories, Burlingame, CA, USA). The cells were examined using a Zeiss Axiovert 100 TV microscope (Carl Zeiss, Thornwood, NY, USA) with a 40x 11.3 NA objective lens equipped with a laser scanning confocal attachment (LSM 410 Carl Zeiss). The staining intensity of cells was quantified as previously described $(16,21)$. Composite fluorescence images were generated and collected by excitation at $488 \mathrm{~nm}$ using an argon/krypton laser (488 nm) as previously described (16). A semi-quantitative estimation based on the relative staining intensity of protein expression was determined for the parental, non-tumorigenic and tumorigenic cell lines. The number of immunoreactive cells ( 30 cells/ field) was counted in 5 randomly selected microscopy fields per sample. Standard errors of the mean are shown in the 
representative figures. Statistical analysis was carried out with the F-test (randomized block) and comparisons between groups with the Bonferroni-t-test with significance at a P-value of $<0.05$.

For evaluation of protein expression by immunoperoxidase staining, exponentially growing cell lines were plated on a glass chamber slide (Nunc Inc.), at a density of $1 \times 10^{4}$ cells $/ \mathrm{ml}$ of medium and allowed to grow for 2-3 days until $70 \%$ confluence (21). The cells were fixed with buffered paraformaldehyde at room temperature, incubated with $1 \% \mathrm{H}_{2} \mathrm{O}_{2}$ in methanol to block endogenous peroxidase and again washed twice with buffer solution. Subsequently, cell cultures were then covered with normal horse serum for $30 \mathrm{~min}$ at room temperature and incubated with the anti-rabbit monoclonal antibody (Santa Cruz Biotechnology) at a 1:500 dilution overnight at $4^{\circ} \mathrm{C}$ and then incubated for $45 \mathrm{~min}$ with diluted biotinylated secondary antibody solution (Vector Laboratories) and Vectastin Elite $\mathrm{ABC}$ reagent (Vector Laboratories) was used. The experiments were repeated thrice in cells with identical passages in vitro. The number of immunoreactive cells (50 cells/fields) was counted in several randomly selected microscopic fields (x400) per sample; 10 fields were counted for each cell line.

Fluorescence-labeled probe preparation for the microarray analysis. The poly(A) mRNA from normal, radiationand estrogen-treated breast cell lines were isolated using QIA-Direct-mRNA Isolation kit (Qiagen, Valencia, CA, USA). Fluorescence-labeled cDNA was prepared from $1 \mu \mathrm{g}$ of each of these poly(A) mRNAs by using oligo dT-primed polymerization and Superscript II reverse transcriptase kit (Life Technologies) in the presence of either Cy3- or Cy5-labeled dCTP following the standard procedure as described (http:// cmgm.stanford.edu/pbrown/protocols.html). The appropriate $\mathrm{Cy} 3$ - and Cy5-labeled probes were pooled and hybridized to the microarray on glass coverslips for $16 \mathrm{~h}$ at $65^{\circ} \mathrm{C}$ and then washed with high stringency for analysis.

\section{Affymetrix HG-U133A Plus 2.0 GeneChip microarray gene} expression analysis. The breast cancer model (Alpha model) consisting of: i) MCF-10F, ii) Estrogen, (iii) Alpha3, iv) Alpha5 and v) Tumor2 cell lines was used to analyze gene expression by the Affymetrix U133A oligonucleotide microarray (Affymetrix, Santa Clara, CA, USA) which contains 14,500 genes. Arrays were quantitatively analyzed for gene expression using the Affymetrix GeneChip Operating software (GCOS) with a dual global scaling option in the Genes@Work software platform of the discovery algorithm SPLASH (structural pattern localization analysis by sequential histograms) with a false discovery rate of 0.05 (26).

\section{Results}

Previous morphological research (17) indicated that the parental MCF-10 and Estrogen cell lines did not exhibit any of the features that characterize malignant cells (anchorageindependent growth in soft agar, invasion and tumor growth in nude mice). In contrast to MCF-10F, the Alpha3 cell line formed colonies in soft agar and invaded but failed to form tumors in immunosuppressed mice. However, the Alpha5 cell line induced mammary gland tumors in animals after cell
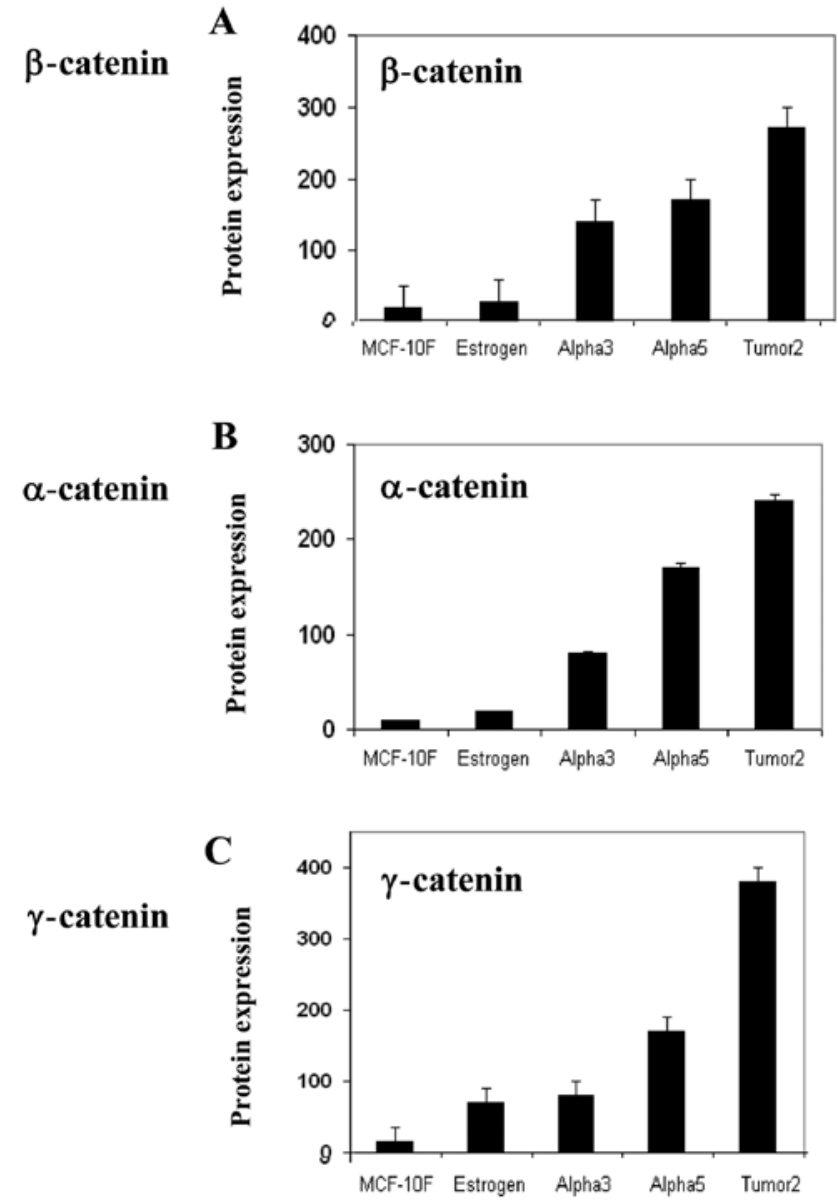

Figure 1. Bars in the histograms represent the average and standard error of (A) $\beta$-catenin (B) $\alpha$-catenin and (C) $\gamma$-catenin protein expression in the MCF-10F, Estrogen, Alpha3, Alpha5 and Tumor2 cell lines as determined by immunofluorescence staining and quantified using confocal microscopy and computer software, which provides the area and the intensity of the staining as described in the text. The primary antibodies used were mouse monoclonal antibodies.

injection. The cell line derived from such tumors was named Tumor2. In the present study, all of these malignant and nonmalignant cell lines were used to analyze the expression of adhesion molecules in breast carcinogenesis.

The immunofluorescence data obtained concerning the relative expression of different adhesion molecules in the MCF-10F, Estrogen, Alpha3, Alpha5 and Tumor2 cell lines are shown in Fig. 1. $\alpha$-catenin, $\beta$-catenin and $\gamma$-catenin expression was significantly $(\mathrm{P}<0.05)$ higher in the Alpha5 and Tumor2 cell lines when compared to the levels in the MCF-10F, Estrogen and Alpha3 cell lines. Representative images of immunoperoxidase and fluorescence staining are presented in Fig. 2. Fig. 3A and $\mathrm{C}$ show the average values of the levels of E-cadherin and integrin $\beta-6$ protein expression in the MCF-10F, Estrogen, Alpha3, Alpha5 and Tumor 2 cell lines as determined by immunoperoxidase staining Fig. 3B and D. Such expression was significantly $(\mathrm{P}<0.05)$ higher in the Alpha5 and Tumor 2 cell lines than that in the MCF-10F, Estrogen and Alpha3 cell lines. However, the Tumor2 cell line had reduced expression levels of E-cadherin and integrin $\beta-6$ protein when compared to that in the Alpha5 cell line, but was higher than that in the Alpha3, Estrogen 


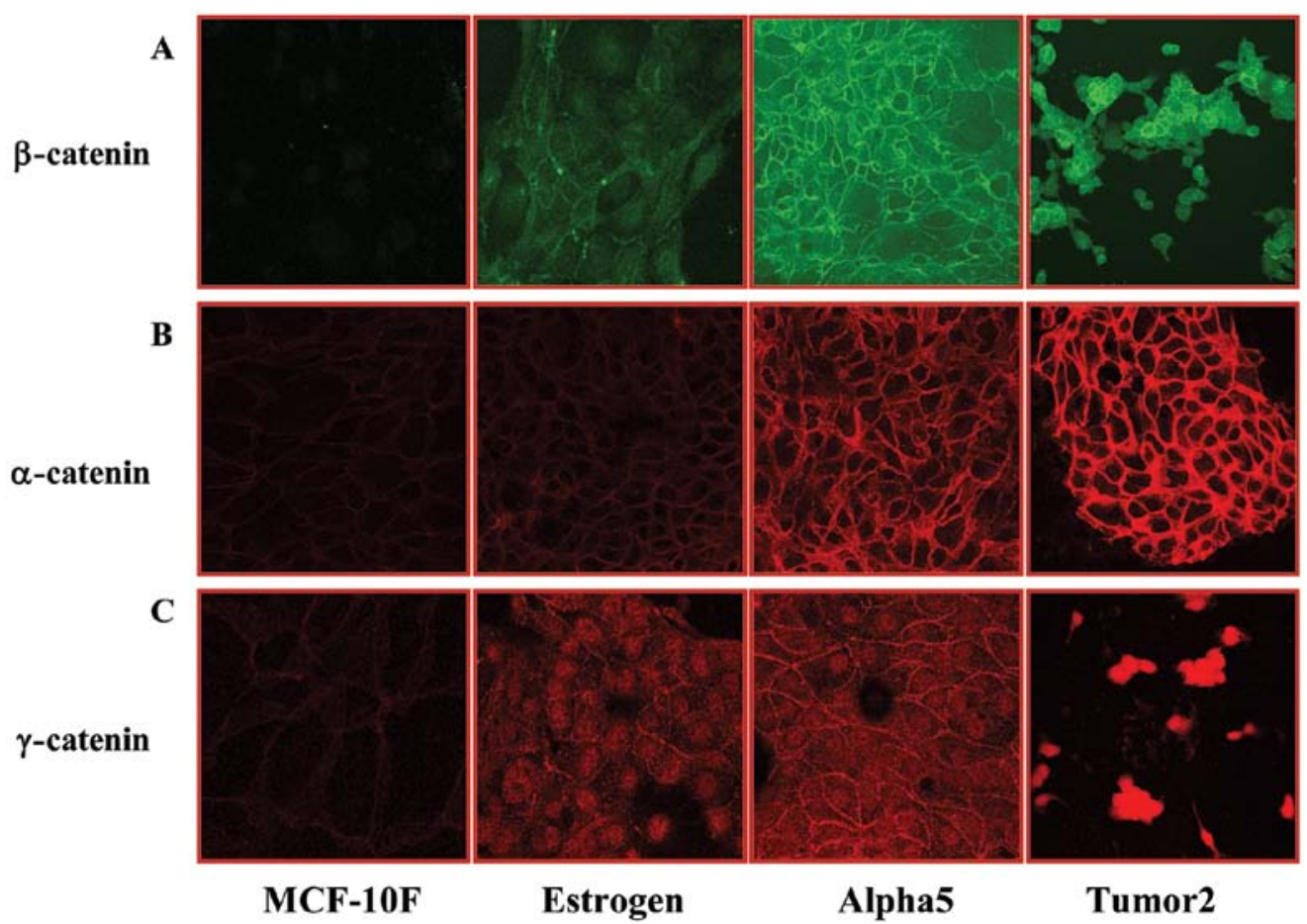

Figure 2. Representative images of (A) $\beta$-catenin (B) $\alpha$-catenin and (C) $\gamma$-catenin protein expression in the MCF-10F, Estrogen, Alpha5 and Tumor2 cell lines using immunofluorescence and immunoperoxidase staining techniques.

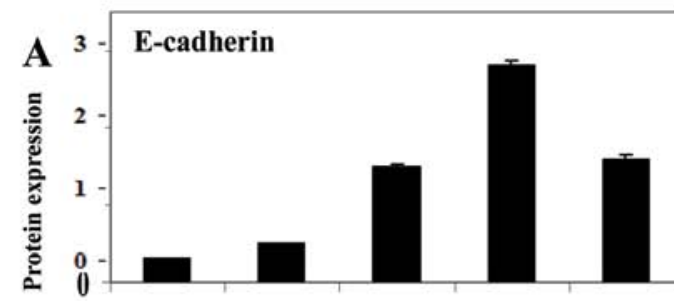

C

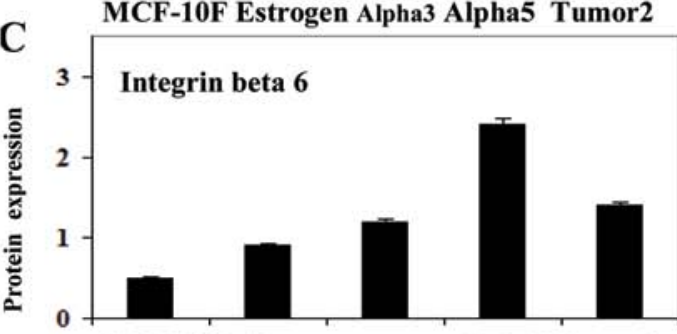

E MCF-10F Estrogen Alpha3 Alpha5 Tumor2

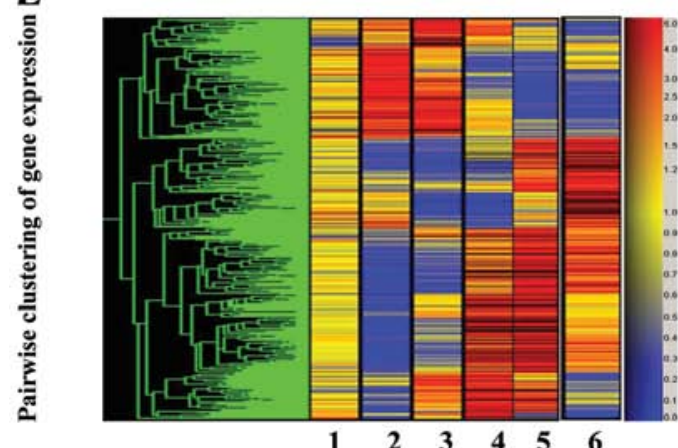

B
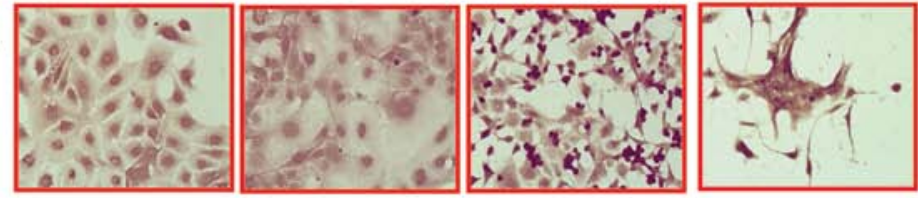

E-cadherin

D

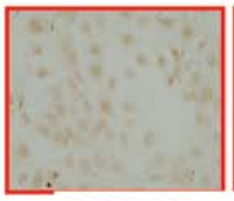

MCF-10F

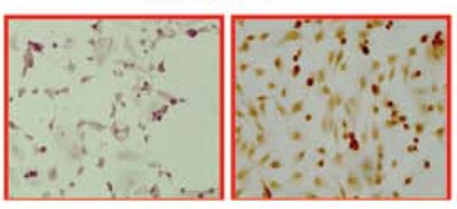

Alpha5

Alpha3

Integrin beta 6

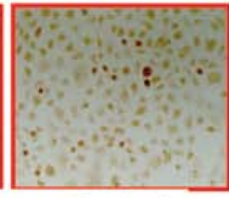

Tumor2

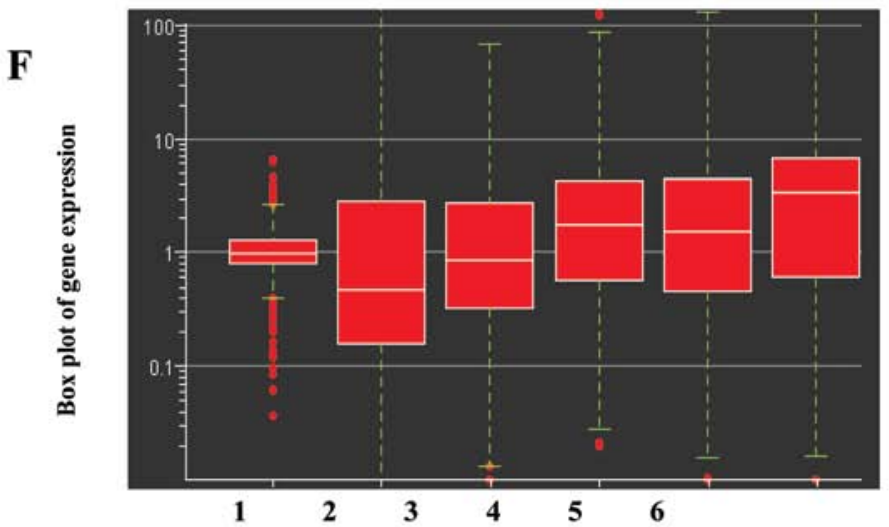

Figure 3. Bars in the histograms represent the average (A) E-cadherin and (C) integrin $\beta 6$ protein expression in the MCF-10F; Estrogen, Alpha3, Alpha5 and Tumor 2 cell lines as determined by peroxidase staining. Representative images of (B) E-cadherin and (D) integrin $\beta 6$ protein expression in the MCF-10F, Alpha3, Alpha5 and Tumor 2 cell lines as stained by peroxidase techniques. The primary antibodies used were mouse monoclonal antibody. (E) Clusterdendrogram of gene expression and $(\mathrm{F})$ fold-change gene expression indicated by a scatter plot of the following pairwise comparative studies of the cell lines: 1, MCF-10F/E; 2, MCF-10F/Alpha3; 3, E/Alpha5; 4, Alpha3/Alpha5; 5, Alpha 3/Tumor2 and 6, Alpha5/Tumor2. 
Table I. Fold-change and pair-wise analysis of differentially expressed genes a breast cancer model.

\begin{tabular}{|c|c|c|c|c|c|c|}
\hline \multirow[b]{3}{*}{ Cell lines } & \multicolumn{6}{|c|}{ Genes } \\
\hline & \multicolumn{2}{|c|}{ E-cadherin } & \multicolumn{2}{|c|}{ Integrin $\beta-6$} & \multicolumn{2}{|c|}{$\mathrm{DSc} 3$} \\
\hline & Fold-change & Regulation & Fold-change & Regulation & Fold-change & Regulation \\
\hline MCF-10F/Estrogen & 1.3 & $\uparrow$ & -1.0 & $\downarrow$ & -1.4 & $\downarrow$ \\
\hline MCF-10F/Alpha3 & -19.9 & $\downarrow$ & 3.6 & $\uparrow$ & -3.2 & $\downarrow$ \\
\hline Estrogen/Alpha5 & -7.3 & $\downarrow$ & -1.2 & $\downarrow$ & 1.6 & $\uparrow$ \\
\hline Alpha3/Alpha5 & 5.6 & $\uparrow$ & -4.7 & $\downarrow$ & 3.6 & $\uparrow$ \\
\hline Alpha3/Tumor2 & 2.6 & $\uparrow$ & 3.1 & $\uparrow$ & 3.6 & $\uparrow$ \\
\hline Alpha5/Tumor2 & 14.3 & 1 & -1.5 & $\downarrow$ & 13.1 & $\uparrow$ \\
\hline
\end{tabular}

$\uparrow$, upregulation; $\downarrow$, downregulation.

and MCF-10F cell lines. The levels of these proteins were similar in the parental MCF-10F and Estrogen cell lines.

Genes that were found to be differentially expressed between the cell lines of the established Alpha model were studied. Cluster-dendrogram and fold changes in gene expression of the cell line model are shown in Fig. 3E and F. Histogram plots showed differential expression of E-cadherin, integrin $\beta-6$ and DSc3 genes in the cell lines as detected by gene chip array. Results of the pairwise comparison of the cell lines examined for the expression of the abovementioned genes are shown in Table I. The following pairs of cell lines were analyzed: MCF-10F/Estrogen (E) (Fig. 3F); MCF-10F/Alpha3; Estrogen/Alpha5; Alpha3/Alpha5; Alpha5/Tumor2; and Alpha 3/Tumor2. Results indicated that the pair-wise comparison did not reveal any alteration in E-cadherin expression between MCF-10F/Estrogen (E) cell lines whereas there was almost a 19- and 7-fold alteration in MCF-10F/Alpha3 and MCF-10F/Alpha5 combinations. Similarly, between Alpha3/Alpha5, there was a 6-fold change in E-cadherin gene expression. Comparison of Alpha3/Tumor2 and Alpha5/Tumor2 cell lines revealed 14- and 3-fold changes in expression, respectively. Comparison between MCF-10F/Alpha3, Alpha3/Alpha5, and Alpha5/Tumor2 showed $\sim 4-, 5$ - and 3-fold changes, respectively in integrin $\beta-6$ gene expression. However, no significant changes in expression were observed in other pairwise combinations. Finally, pairwise comparison of gene expression between MCF-10F/ Alpha3, Alpha3/Alpha5 and Alpha5/Tumor2 showed changes of 3- to 4-fold whereas comparison of the Alpha3/Tumor2 cell lines revealed a 13-fold change in expression for the DSc3 gene. MCF-10F/Estrogen (E) and MCF-10F/Alpha5 combinations showed no significant alterations in gene expression.

\section{Discussion}

Our previous study indicated that the combined treatment of ionizing radiation and estrogen yielded different stages in a malignantly transformed breast cancer cell model system, which we termed the Alpha model system (17). Utilizing this model system, altered expression of different cell adhesion molecules was detected in the parental, non-tumorigenic and malignantly transformed cell lines originally derived from the parental MCF-10A cell line. Results of this study indicated that some of the cell adhesion molecules may have prognostic/ diagnostic significance for breast carcinogenesis.

Expression levels of $\alpha$-catenin, $\beta$-catenin and $\gamma$-catenin were significantly greater in the Alpha5 and Tumor 2 cell lines when compared to these levels in the MCF-10F, Estrogen and Alpha 3 cell lines. $\beta$-catenin appears to be a critical component of a complex signal transduction pathway that regulates the central process of cellular proliferation and differentiation. The intracellular $\beta$-catenin level was found to be regulated by its association with the adenomatous polyposis coli tumorsuppressor protein and GSK-3- $\beta(10)$. Consistent with our study, a previous study also showed an increased level of $\beta$-catenin in human bronchial epithelial cells transformed by treatment with the tobacco-specific nitrosamine, 4-(methylnitrosamino)1-(3-pyridyl)-1-butatone (27). Other studies reported that catenins, particularly when expressed in the cytoplasm with the E-cadherin complex, are sensitive prognostic markers for invasive breast cancer $(3,7)$ since the E-cadherin/ $\alpha$-catenin complex is capable of modulating cell-cell and cell-matrix adhesive properties $(3,7,11)$. In support of this finding, the E-cadherin/ $\alpha$-catenin complex has also been shown to modulate cell-cell and cell-matrix adhesive properties of invasive colon carcinoma cells (3). The E-cadherin-catenin complex is also the target of many growth factors and hormone-dependent signaling pathways that regulate its function and expression (24). E-cadherin expression correlates with poor survival in breast carcinoma even though it is strongly expressed in both luminal and myoepithelial cells (28). Adherent junctions and desmosomes are characteristic of epithelial cells. In the present study, we found that E-cadherin protein expression was higher in the malignantly transformed Alpha5 cell line than that in the parental MCF-10F cell line. However, reduced E-cadherin expression was found in the xenograft derived Tumor 2 cell line when compared to that in Alpha5 although the precise reason for this is not clear. Both E-cadherin and integrin $\beta-6$ proteins were overexpressed in Alpha5 when compared to the 
protein levels in the MCF-10F, Estrogen and Alpha3 cell lines as determined by immunoperoxidase staining. Integrin $\beta-6$ and the DSc3 genes were expressed at higher levels in both the Alpha3 and Alpha5 cell lines. Gene expression analysis identified several of the adhesion molecules that were differentially expressed in carcinogenesis. Reduced E-cadherin expression similar to that observed in the Tumor 2 cell line probably indicates the loss of the epithelial phenotype. Reduced E-cadherin is common in many breast carcinomas and it is frequently lost in infiltrating lobular carcinomas which otherwise clearly exhibit an epithelial phenotype (29).

In previous studies, cell adhesion was also analyzed by $\beta$-catenin protein expression and was also found higher in cells treated with parathion alone and estrogen combined with parathion in comparison to control and estrogen-treated cells. The function of the cadherin-catenin system in cell adhesion as well as intracellular signaling appears to be subjected to multifactorial control by a variety of different mechanisms. $\beta$-catenin had a similar reaction in the presence of parathion alone and combined with estrogen in comparison to the control and in the presence of estrogen. However, it seems that estrogens did not play a role in this pesticide-induced model mediated by the cadherin-catenin complex since both substances had equal effect. This complex seems to initiate signaling events implicated in differentiation and growth control. Other studies have indicated that the E-cadherin-catenin complex is the target of many growth factors and hormone-dependent signaling pathways which regulate its function and expression $(25,30)$. Other authors have found that catenin, particularly when it is expressed in the cytoplasm, seems to be a very sensitive prognostic marker with the E-cadherin complex in invasive breast cancer $(1,3,9,10)$. It may have invasive capabilities since a possible role of the E-cadherin/ $\alpha$-catenin complex in modulating cell-cell and cell-matrix adhesive properties of invasive colon carcinoma cells has been reported (25). There is evidence that changes in the shape of the epithelial surface are features of the cell transformation of epithelial cells. Our results suggest that aberrant expression of $\beta$-catenin may be involved in tumor metastasis.

Several genes involved in adhesion function such as E cadherin, integrin $\beta-6$, and Dsc3 were found through gene expression microarray studies. E-cadherin was not significantly different when the MCF-10F and Estrogen cell lines were compared. Differential gene expression has been reported in the literature between luminal and myoepithelial cells when DSc2 and DSc3 were compared with the DSc3 gene expression solely found in myoepithelial population (30). Thus, the expression profile of genes, particularly in conjunction with other markers, can help to distinguish between luminal and myoepithelial cells. In the present study, there was a decrease in DSc3 gene expression. Integrin signaling is a well known requirement for the complex process of metastasis which seems to occur through a series of steps that involve local tissue invasion, intravasation and survival in colonization and circulation $(14,30)$. The $\alpha-6 \beta-4$ integrin complex is strongly expressed in myoepithelial cells but not in luminal epithelium. Expression of desmosomal cadherins is largely confined to epithelial cells. Integrin $\beta-6$ protein expression was significantly greater in Alpha5 than in the MCF-10F and estrogen cell lines while the Tumor 2 cell line exhibited a decreased protein expression when compared with Alpha5. The $\alpha-6 \beta-4$ integrin complex is strongly expressed by myoepithelial cells but not in luminal epithelium $(13,30)$.

Results of this study suggest that environmental agents (e.g., ionizing radiation) in the presence of estrogen can drastically affect human breast cell adhesion phenomena thereby promoting or supporting the molecular events of the process of cellular transformation. Future studies are required to verify whether the altered expression of cell adhesion molecules precedes or accompanies the cellular transformation process. Elucidation of the precise role of cell adhesion molecules in carcinogenic events may be helpful to assess their prognostic/diagnostic significance for breast carcinogenesis in clinical settings.

\section{Acknowledgements}

The support provided by FONDECYT \#1120006 (G.M.C.) and MINEDUC Universidad de Tarapacá (G.M.C.) is greatly appreciated. We also thank Dr Manikandan Jayapal and Dd Praksah Hande of the National University of Singapore for the analysis of the Affymetrix microarray data.

\section{References}

1. Pierceall WE, Woodard AS, Morrow JS, Rimm D and Fearon ER: Frequent alterations in E-cadherin and alpha- and beta-catenin expression in human breast cancer cell lines. Oncogene 11: 1319-1326, 1995.

2. Ozawa M, Terada $\mathrm{H}$ and Pedraza $\mathrm{C}$ : The fourth armadillo repeat of plakoglobin (gamma-catenin) is required for its high affinity binding to the cytoplasmic domains of E-cadherin and desmosomal cadherin Dsg2, and the tumor suppressor APC protein. J Biochem 118: 1077-1082, 1995

3. Breen E, Steele G Jr and Mercurio AM: Role of the E-cadherin/ alpha-catenin complex in modulating cell-cell and cell-matrix adhesive properties of invasive colon carcinoma cells. Ann Surg Oncol 2: 378-385, 1995.

4. Knudsen KA, Soler AP, Johnson KR and Wheelock MJ: Interaction of $\alpha$-actinin with the cadherin/catenin cell-cell adhesion complex via $\alpha$-catenin. J Cell Biol 130: 67-77, 1995.

5. Sacco PA, McGranahan TM, Wheelock MJ and Johnson KR: Identification of plakoglobin domains required for association with N-cadherin and $\alpha$-catenin. J Biol Chem 25: 20201-20206, 1995.

6. Wahl JK, Sacco PA, McGranahan-Sadler TM, Sauppé LM, Wheelock MJ and Johnson KR: Plakoglobin domains that define its association with the desmosomal cadherins and the classical cadherins: identification of unique and shared domains. Cell Sci 109: 1143-1154, 1996.

7. Takayama T, Shiozaki H, Shibamoto S, Oka H, Kimura Y, Tamura S, Inoue M, Monden T, Ito F and Monden M: Betacatenin expression in human cancers. Am J Pathol 148: 39-46, 1996.

8. Salomon D, Sacco PA, Roy SG, Simcha I, Johnson KR, Wheelock MJ and Ben-Ze'ev A: Regulation of beta-catenin levels and localization by over-expression of plakoglobin and inhibition of the ubiquitin-proteasome system. J Cell Biol 139: 1325-1335, 1997.

9. Lim SC and Lee MS: Significance of E-cadherin/ $\beta$-catenin complex and cyclin D1 in breast cancer. Oncol Rep 9: 915-928, 2002 .

10. Nakopoulou L, Gakiopoulou-Givalou H, Karayiannakis AJ, Giannopoulou I, Keramopoulos A, Davaris P and Pignatelli M: Abnormal $\alpha$-catenin expression in invasive breast cancer correlates with poor patient survival. Histopathology 40: 536-546, 2002.

11. Tagliabue E, Ghirelli C, Squicciarini P, Aiello P, Colnaghi MI, Menard S: Prognostic value of alpha 6 beta 4 integrin expression in breast carcinomas is affected by laminin production from tumour cells. Clin Cancer Res 4: 407-410, 1998. 
12. Stingl J, Eaves CJ, Kuusk U and Emerman JT: Phenotypic and functional characterization in vitro of a multipotent epithelial cell present in the normal adult human breast. Differentiation 63: 201-213, 1998

13. Jones C, Nonni AV, Fulford L, Merrett S, Chaggar R, Eusebi V and Lakhani SR: CGH analysis of ductal carcinoma of the breast with basaloid/ myoepithelial cell differentiation. Br J Cancer 85: 422-427, 2001

14. Friedrichs K, Ruiz P, Franke F, Gille I, Terpe HJ and Imhof BA: High expression level of $\alpha-6$ integrin in human breast-carcinoma is correlated with reduced survival. Cancer Res 55: 901-906, 1995.

15. Calaf GM and Russo J: Transformation of human breast epithelial cells by chemical carcinogens. Carcinogenesis 14: 483-492, 1993.

16. Calaf GM and Hei TK: Establishment of a radiation- and estrogen-induced breast cancer. Carcinogenesis 21: 769-776, 2000.

17. Soule HD, Maloney TM, Wolman SR, Peterson WD Jr, Brenz R McGrath CM, Russo J, Pauley RJ, Jones RF and Brooks SC: Isolation and characterization of a spontaneously immortalized human breast epithelial cell line MCF-10. Cancer Res 50: 6075-6086, 1990.

18. Calaf GM, Russo J and Alvarado ME: Morphological phenotypes in neoplastic progression of benz (alpha) pyrene-treated breast epithelial cells. J Submicrosc Cytol Pathol 32: 535-545, 2000.

19. Calaf GM and Hei TK: Oncoprotein expressions in human breast epithelial cells transformed by high-LET radiation. Int J Radiat Biol 77: 31-40, 2001.

20. Calaf G, Alvarado $M$ and Hei TK: Beta catenin is associated with breast cancer progression in vitro. Int J Oncol 26: 913-921, 2005.
21. Calaf G, Roy D and Hei TK: Immunochemical analysis of protein in breast epithelial cells transformed by estrogens and high linear energy transfer (LET) radiation. Histochem Cell Biol 124: 261-274, 2005.

22. Calaf G, Alvarado ME and Hei TK: Oncoprotein expression and morphological phenotypes of human breast epithelial cells transformed by c-Ha-ras oncogene. Oncol Rep 14: 885-893, 2005.

23. Calaf GM and Roy D: Gene expression signature of parathiontransformed human breast epithelial cells. Int J Mol Med 19: 741-750, 2007.

24. Calaf GM and Roy D: Gene and protein expressions induced by 17 beta estradiol and parathion in cultured breast epithelial cells. Mol Med 13: 255-265, 2007.

25. Calaf GM and Roy D: Cell adhesion proteins altered by $17 \beta$ estradiol and parathion in breast epithelial cells. Oncol Rep 19: $165-169,2008$.

26. Califano A: SPLASH: Structural pattern localization analysis by sequential histograms. Bioinformatics 16: 341-357, 2000.

27. Zhou H, Calaf GM and Hei TK: Malignant transformation of human bronchial epithelial cells with the tobacco-specific nitrosamine, 4-(methylnitrosamino)-1-(3-pyridyl)-1-butatone. Int J Cancer 106: 821-826, 2003

28. Peralta Soler A, Knudsen KA, Salazar H, Han AC and Keshgegian AA: P-cadherin in breast carcinoma indicates poor survival. Cancer 86: 1263-1272, 1999.

29. Moll R, Mitze M, Frixen UH, Birchmeier W: Differential loss of E-cadherin expression in infiltrating ductal and lobular breast carcinoma. Am J Pathol 143: 1731-1742, 1993.

30. Gordon LA, Mulligan KT, Maxwell-Jones H, Adams M, Walker RA and Jones JL: Breast cell invasive potential relates to the myoepithelial phenotype. Int J Cancer 106: 8-16, 2003. 